九州大学学術情報リポジトリ

Kyushu University Institutional Repository

\title{
Compression and recovery micro-mechanisms in flexible graphite
}

Toda, Hiroyuki

Department of Mechanical Engineering, Toyohashi University of Technology

Tsubone, Kodai

Department of Mechanical Engineering, Toyohashi University of Technology

Shimizu, Kazuyuki

Department of Mechanical Engineering, Toyohashi University of Technology

Uesugi, Kentaro

Japan Synchrotron Radiation Research Institute

他

http://hdl. hand le. net/2324/1807802

出版情報 : Carbon. 53，pp. 184-191，2013-08-01

バージョン：

権利関係 : 


\section{Compression and recovery micro-mechanisms in flexible graphite}

Hiroyuki Toda $^{* a}$, Kodai Tsubone ${ }^{\mathrm{a}}$, Kazuyuki Shimizu ${ }^{\mathrm{a}}$, Kentaro Uesugi ${ }^{\mathrm{b}}$, Akihisa

Takeuchi $^{\mathrm{b}}$, Yoshio Suzuki ${ }^{\mathrm{b}}$, Mitsuru Nakazawa ${ }^{\mathrm{c}}$, Yoshimitsu Aoki ${ }^{\mathrm{c}}$ and Masakazu

Kobayashi $^{\mathrm{a}}$

${ }^{a}$ Department of Mechanical Engineering, Toyohashi University of Technology, 1-1, Hibarigaoka, Tempaku, Toyohashi, Aichi 441-8580, Japan

b Japan Synchrotron Radiation Research Institute, 1-1-1, Kouto, Mikazuki-cho, Sayo-gun, Hyogo 679-5198, Japan

${ }^{c}$ Electronics \& Electrical Engineering, Keio University, 3-14-1, Hiyoshi, Kohoku-ku, Yokohama, Kanagawa 223-8522, Japan

* Corresponding author. Tel/Fax: +81 532446697 / +81 532 446690. E-mail address: toda@me.tut.ac.jp (H. Toda) 


\begin{abstract}
Utilization of synchrotron X-ray microtomography has enabled the virtual reconstruction of invisible microstructural units and their deformation during the compaction, compression and recovery of flexible graphite. Particles artificially affixed, in advance, to the surface of a given microstructural unit were connected by plane triangles to create a 3D mesh in the reconstruction. High density 3D mapping of strain and displacement has also been achieved, by tracing the physical displacement of the surface particles. Micro-mechanisms of compression and recovery behavior in flexible graphite have been examined involving the direct observations and measurements. The microstructural unit was found to be composed of thin expanded graphite discs with slightly misaligned basal planes of graphite. Highly localized deformation of a thin expanded graphite disc was observed during the compression and recovery processes, suggesting strong interaction with surrounding expanded graphite discs. The macroscopic compression behavior of flexible graphite is attributable to a combination of bending and thickness reduction/recovery of the discs. It has, however, been shown that only the thickness recovery of the individual discs, due to the presence of internal entrapped air, accounts for the macroscopic recovery behavior of flexible graphite.
\end{abstract}




\section{Introduction and background}

Flexible graphite is a flexible thin sheet made by compacting expanded graphite powder [1]. The expanded graphite is industrially derived by expanding acid-intercalated graphite several hundred times, into a structure composed of highly porous worm-like particles (hereinafter called worm) [1, 2]. The expanded graphite exhibits an accordion-like internal structure, resulting in excellent compression and recovery behavior even at high temperatures [1], and making flexible graphite an important sealing and gasketing material [3].

Micro-mechanisms of compression and recovery behavior in flexible graphite have been investigated, to date, with simplified model analysis and/or indirect observations or measurements [4-8]. The model analysis by Leng et al. assumed that the compression and recovery behavior of flexible graphite was associated with the internal pressure of air trapped inside the cavities in the expanded graphite worm [4], whereas Dowell et al. discounted the contribution of such encapsulated air, and concluded, based on surface and cross-sectional observation, that the compression behavior of flexible graphite is attributable to the flexing of disc-shaped expanded graphite with slightly misaligned basal planes of graphite [5]. It has, in any case, been difficult to determine the precise relationship between the relevant microstructure and macroscopic material behavior, due to the lack of direct observation and measurement in conventional experimental approaches. Since flexible graphite essentially consists of brittle thin graphite films, traditional metallographic sample preparation causes extensive damage, hindering both morphological characterization and identification of the relevant microstructural features. 
The objective of the present study is to resolve the controversy over the micro-mechanisms of compression and recovery behavior in flexible graphite. State-of-the-art synchrotron X-ray microtomography (XMT) is employed for this purpose. The microstructural tracking technique readily enables the visualization of microstructural features invisible even to X-ray detection [9-11], providing a unique opportunity to quantitatively analyze the compression and recovery behavior of flexible graphite at a microscopic scale; a clear improvement on the conventional procedures of the current literature, which involve only macroscopic or indirect evaluations in this respect.

\section{Experimental methods}

\subsection{Tomographic imaging}

A high-resolution projection-type XMT experiment was performed using the BL20XU X-ray imaging beamline of the SPring-8 synchrotron radiation facility. An in-situ loading rig specially designed for this experiment, schematically shown in Fig. 1, was positioned approximately $80 \mathrm{~m}$ from the X-ray source. A monochromatic X-ray beam with photon energy of $25 \mathrm{keV}$, generated by a double crystal monochromator, was used. A $4000(\mathrm{H}) \times 2624(\mathrm{~V})$ element CCD camera, in $2 \times 2$ binning mode, and a $\mathrm{Lu}_{2} \mathrm{SiO}_{5}: \mathrm{Ce}$ single crystal scintillator, were used as a detector. The image detector was positioned $55 \mathrm{~mm}$ behind the loading rig, which generated a pair of fringes $(1.65 \mu \mathrm{m}$ in width of each fringe) at each edge of the three-dimensional (3D) images, thereby enhancing the detectability of features otherwise invisible in ordinary absorption-contrast X-ray imaging. A total of 1500 radiographs, scanning 180 degrees in 
0.12-degree increments, were obtained. A cylindrical region of interest (roughly 1000 $\mu \mathrm{m}$ in diameter and $656 \mu \mathrm{m}$ in height) was captured on the CCD camera. Image slices were reconstructed from a series of projections based on the conventional filtered backprojection algorithm. An isotropic voxel with a $0.5 \mu \mathrm{m}$ edge was achieved in the reconstructed slices. The grey value in each dataset was calibrated such that the linear absorption coefficient of 0 to $\mu_{\max } \mathrm{cm}^{-1}$ ( $\mu_{\max }$ varied between 35 and 80, depending on the degree of compaction) fell within an 8-bit gray scale range between 0 and 255 .

The in-situ loading rig allowed expanded graphite powder, which is made of expanded graphite worms, to be scanned during its compaction, compression and recovery processes. First, half of the total amount of raw expanded graphite powder necessary for the experiment was poured into the cavity of the in-situ loading rig, and one expanded graphite worm was then placed on the as-poured raw expanded graphite powder. The first tomography scan was performed at this stage, without loading. Since the individual expanded graphite worm would not be identifiable after compaction, commercial $\mathrm{ZnO}$ and $\mathrm{WC}$ particles were affixed to the surface of the expanded graphite worm with a minute amount of organic binder, in advance, as marker particles. The $\mathrm{ZnO}$ and WC particles were roughly 5 and $1 \mu \mathrm{m}$ in average diameter, respectively. The particles were sufficiently opaque under X-ray irradiation, whereas the organic binder was transparent and stable under X-ray irradiation. The remaining half of the total raw expanded graphite powder was then poured into the cavity, and a plunger was inserted into the cavity to compact the raw expanded graphite powder to a thickness of $2 \mathrm{~mm}$. The density of the compacted expanded graphite powder was approximately $1 \mathrm{~g} / \mathrm{cm}^{3}$ at this stage, which corresponds to the typical density of commercially available flexible graphite sheets $[2,4,12]$. Four scans were performed during the initial compression 
process; and seven subsequent scans were performed during further compression of $30 \%$ (with reference to the original density of $1 \mathrm{~g} / \mathrm{cm}^{3}$ ), at approximately $50 \mu \mathrm{m}$ intervals in plunger displacement. The initial four scans and the seven latter scans were assumed to reflect the industrial fabrication process of a flexible graphite sheet and subsequent compression during its practical use, respectively under simulation loads. Therefore the initial four scans and the subsequent seven scans are hereinafter called "compaction process" and "compression process", respectively to indicate their industrial meanings. Finally, three scans were performed as the compression was decreased at approximately $50 \mu \mathrm{m}$ intervals. The variations in sample height, measured in projected images, are summarized in Fig. 2.

\subsection{Image analysis}

Precise image registration was performed before the particles were tracked, using a transformation matrix, which minimizes the sum of the distances between identical particles captured at neighboring scan steps. The particles were then tracked, throughout loading, using the 3D microstructural tracking technique, which consists of the matching parameter method with the modified spring model $[9,10]$. The coefficients $\alpha, \beta$ and $\gamma$, and the search range in the matching probability parameter, all as in references 9 and 10 , were determined to be $0.8,0.1,0.1$, and 65 to $125 \mu \mathrm{m}$, respectively, after systematically searching for the optimum conditions in a preliminary trial. 3D strain and displacement mappings were then performed, based on the physical displacement of the tracked marker particles $[9,10]$. It has been reported that the error in the $3 \mathrm{D}$ microstructural tracking technique is significantly smaller than that of the spatial resolution of 3D images, which is about $1 \mu \mathrm{m}$ in the case of the projection-type 
XMT) [10].

The expanded graphite worm affixed with $\mathrm{ZnO}$ and $\mathrm{WC}$ particles (which would be unidentifiable in the flexible graphite without these particles) was reconstructed as a polygon mesh. Groups of segmented particles, which are pixels clustered into salient image regions corresponding to individual particles after image segmentation, located on the surface of the expanded graphite worm were considered as vertices, and connected by plane triangles to create 3D meshes without overlapping or gaps, as shown in Fig. 3. Such polygon meshes represent the outer contours of the expanded graphite worm. The microstructural tracking technique enables visualization of the deformation behavior of the expanded graphite worm, throughout the in-situ loading experiment; and the combination of the morphological information on the expanded graphite worm and the 3D strain/displacement mappings offers a direct method of evaluating the fundamental compression and recovery mechanisms of flexible graphite at a microstructural unit scale.

\section{Microstructural unit}

After the first loading in the compaction process, the expanded graphite worms could be roughly observed in $3 \mathrm{D}$, without employing the polygon mesh technique shown in Fig. 3. Fig. 4, for example, shows a 3D perspective view of an expanded graphite worm, and a portion of the expanded graphite worm that has been cut away with a clip plane, which cuts away the portion of the expanded graphite worm on one side of the plane; both shown at loading steps 0 and 1 . The expanded graphite exhibits microscopic disc-like shape (hereinafter called microdisc), with a complex layered 
internal structure. It may be observed that the basal planes of graphite are almost parallel to the ligament (i.e., thin graphite internal layers), and are slightly misaligned with respect to each other due to irregular overlapping with neighboring microdiscs. The thickness of the microdisc varies greatly from place to place, ranging from roughly 40 to $110 \mu \mathrm{m}$ in Fig. 4 (c). Significant bending of the microdisc is clearly observable after the first compaction step (Fig. 4 (d)), and the bending seems to be closely associated with thickness reduction at these early compaction stages. The layered internal structure is sometimes discontinuous, and its pre-loading interlayer thickness appears to range from 5 to $10 \mu \mathrm{m}$ at this magnification.

After the initial loading steps, the microdiscs became invisible to X-ray detection, and therefore polygon meshes were used for the continued morphological evaluation of the microdisc of interest, as in Fig. 5. Fig. 5 (a), corresponding to Fig. 4 (a), shows the microdisc with considerable inclination from the horizontal axis, which is significantly reduced during the compaction process shown in Fig. 5 (a) to (d). After this, no significant change is observable in its morphology in Fig. 5, except that the asperity, most notable on the right side of the microdisc, almost disappears through flattening. In order to confirm the morphological changes in the compression process, the microdisc images just before and after the compression process have been superposed in Fig. 6. Both bending and thickness reduction are observable in Fig. 6, and there is buckling displacement of approximately $15 \mu \mathrm{m}$, at the left edge, between the two loading steps. The respective bending and thickness reduction behaviors are quantitatively characterized in the following sections.

\section{Bending of the microdisc}


Fig. 7 shows 3D displacement vector distributions viewed from an oblique direction, representing the compaction, compression and recovery processes, respectively. Overall, the microdisc shows bending (as in Fig. 7 (a)), along with substantial flattening (as in Figs. 5 (a) and (b)), during the compaction process. The distribution of 3D displacement vectors is however complex, suggesting strong interaction with numerous surrounding microdiscs during the compaction process. Three-point bending deformation is clearly observable during the compression process (Fig. 7 (b)), in a reverse displacement direction to that of the compaction process. However, in the recovery process, no obvious deformation pattern is observed, with upward and downward displacements alternatively distributed in a complex manner. In-plane equivalent strain distributions on virtual cross-sections (Fig. 8) also indicate more extensive deformation during the compaction process, as well as a subsequent transition to highly localized deformation during the compression and recovery processes. Notably, in the recovery process especially, the strain is even more localized, supporting the more localized displacement shown in Fig. 7 (c).

\section{Thickness reduction and recovery of the microdisc}

In order to evaluate the thickness reduction and recovery behavior of the microdisc, inter-particle spacing between particles located on the upper and lower surfaces of the microdisc was measured. Particles located within $50 \mu \mathrm{m}$ of each other were paired for this thickness measurement. Fig. 9 shows the thickness reduction and recovery behavior, as normal strain in the $z$ direction (i.e., the compression direction). 
Note that normal strain is opposite in sign for the compression and recovery processes. It is interesting to note that the thickness reduction is relatively uniform especially over the upper right half of the microdisc, during the compression process. Although the extent of thickness recovery observed in the recovery process appears roughly proportional to that of thickness reduction in the compression process, more significant local thickness variation is observed in the recovery process.

It is reasonable to assume that the recovery behavior is accompanied by lateral contraction, especially since the internal pressure of air trapped in the cavities of microdiscs governs the recovery phenomenon. In Fig. 10, in-plane displacement is magnified and superposed on the projected microdisc image viewed from directly above. Relatively uniform centripetal displacements are clearly observable during the recovery process. Poisson's ratio, calculated from the average lateral displacement, was approximately 0.04 . It is well-known that the compressibility of a porous solid derives from the mechanism of volume change in the internal pores during deformation. Thus, a Poisson's ratio equal to zero is acceptable in the case of a porous solid without encapsulated air (i.e., an open-cell foam) [13]. Although the Poisson's ratio of 0.04 is relatively low, it would appear to suggest that there is some contribution from the air trapped in the microdisc cavities to the compression and recovery phenomena of flexible graphite. Given that only incompressible gas is responsible for the universal Poisson deformation of the microdisc, a Poisson ratio is exactly 0.5. If open-cell regions and closed cell regions are uniformly mixed in the microdisc and the macroscopic Poisson's ratio follows the rule of mixture, the total volume fraction of closed-cell regions is calculated to be $8 \%$. It can be inferred that this low fraction allows for further microstructural control to enhance the recovery behavior of microdiscs. 


\section{Relationship between microstructure and macroscopic recovery behavior}

In the present study, macroscopic normal strain in the compression and recovery direction was determined, along with variations in sample thickness. As shown in Figs. 7, 9 and 10, the combined effect of the internal pressure of air trapped in the cavities and the bending of microdiscs accounts for the macroscopic compression and recovery behavior of flexible graphite. The contribution of the thickness reduction/recovery of microdiscs was demonstrated as a form of strain in Fig. 9, however the relationship between microdisc bending and the macroscopic recovery behavior of flexible graphite is not as clear. The latter would appear to be governed by multiple interactions among neighboring microdiscs, causing remarkably localized and complex microdisc deformation. In Fig. 11, microdisc strain generated by microdisc bending has been determined by subtracting microdisc strain due to the thickness reduction/recovery of microdiscs from the macroscopic strain of the sample. It is interesting to note that the bending deformation of microdiscs appears to be saturated during the first half of the compression process, and is not observed again throughout the recovery process. The recovery of flexible graphite is therefore attributable solely to the thickness recovery of the microdiscs. It may be inferred, from Fig. 11, that if macroscopic compression is terminated at a lower applied displacement level, microdisc bending might exert a negligible influence on the macroscopic recovery behavior, since such microdisc bending is apt to occur at a lower applied displacement, before the onset of microdisc thickness reduction. The contribution of microdisc bending may therefore be associated with the level of internal gas pressure. 
There were two important competing factors in the relationships between microstructure and macroscopic recovery behavior - thickness reduction and bending of the microdisc, which were not completely alternative and therefore differed from the past simplified model analyses and the indirect observations or measurements introduced in section 1 [4-8]. The practically important recovery process can however be associated with the microdisc thickness reduction. Although the microdisc thickness reduction is not attributable to the existence of internal gas pressure with conclusive evidence, it can be inferred that the control of raw graphite and its acid-intercalation process and / or the expansion process would be of crucial importance in realizing superior sealing and gasketing material.

\section{Conclusion}

The XMT technique was employed to observe the compaction, compression and recovery behavior of flexible graphite. This approach enabled the reconstruction of $3 \mathrm{D}$ renderings of the deformation of a microstructural unit, in the form of 3D polygon meshes (representing an expanded graphite microdisc) with a reasonably high spatial resolution (approximately $1 \mu \mathrm{m}$ ). The major focus of the in-situ experiment was on determining the dominant recovery mechanism in flexible graphite (i.e., bending and/or thickness recovery of microdisc-shaped expanded graphite). To obtain conclusive evidence for this determination, high density 3D mapping of strain and displacement was employed, by tracking surface particles affixed to a specific microdisc. Although the macroscopic compression behavior of flexible graphite was attributable to both the bending and the thickness reduction/recovery of microdiscs, the macroscopic recovery 
behavior of flexible graphite was attributable only to the thickness recovery of the microdiscs. In the light of the findings reported here, it may be inferred, from a practical point of view, that the closed-cell inter-layer structure and internal gas pressure of expanded graphite microdiscs are of crucial importance in controlling the recovery properties of flexible graphite.

\section{Acknowledgements}

This work was undertaken partly with the support of a Grant-in-aid for Scientific Research from JSPS through subject No. 20246102. The synchrotron radiation experiments were performed with the approval of JASRI through proposal Nos. 2010B1234 and 2011A1498.

\section{References}

[1] Celzard A, Marêché JF, Furdin G. Modelling of exfoliated graphite. Progress in Materials Science 2005; 50(1):93-179.

[2] Reynolds RA III, Greinke RA. Influence of expansion volume of intercalated graphite on tensile properties of flexible graphite. Carbon 2001; 39(3):479-481.

[3] Chung DDL. Flexible graphite for gasketing, adsorption, electromagnetic interference shielding, vibration damping, electrochemical applications, and stress sensing. J Mater Eng Perf 2000; 9(2):161-163.

[4] Leng Y, Gu J, Cao W, Zhang T-Y. Influences of density and flake size on the mechanical properties of flexible graphite. Carbon 1998; 36(7-8):875-881. 
[5] Dowell MB, Howard RA. Tensile and compressive properties of flexible graphite foils. Carbon 1986; 24(3):311-323.

[6] Celzard A, Schneider S, Marêché JF. Densification of expanded graphite. Carbon 2002; 40(12):2185-2191.

[7] Gu J, Leng Y, Gao Y, Liu H, Kang F, Shen W. Facture mechanism of flexible graphite sheets. Carbon 2002; 40(12): 2169-2176.

[8] Kobayashi M, Toda H, Takeuchi A, Uesugi K, Suzuki Y. Three-dimensional evaluation of the compression and recovery behavior in a flexible graphite sheet by synchrotron radiation microtomography. Mater Charact 2012; 69:52-62.

[9] Kobayashi M, Toda H, Kawai Y, Ohgaki T, Uesugi K, Wilkinson DS, Kobayashi T, Aoki Y, Nakazawa M. High-density three-dimensional mapping of internal strain by tracking microstructural features. Acta Mater 2008; 56(1):2167-2181.

[10] Toda H, Maire E, Aoki Y, Kobayashi M. Three-dimensional strain mapping using in situ X-ray synchrotron microtomography. J Strain Anal Eng Des 2011; 46:549-561.

[11] Toda H, Minami K, Koyama K, Ichitani K, Kobayashi M, Uesugi K, Suzuki Y. Healing behavior of preexisting hydrogen micropores in aluminum alloys during plastic deformation. Acta Mater 2009; 57(15):4391-4403.

[12] Chugh R, Chung DDL. Flexible graphite as a heating element. Carbon 2002; 40(13):2285-2289.

[13] Gioux G, McCormack TM, Gibson LJ. Failure of aluminum foams under multiaxial loads. Int J Mech Sci 2000;42(6):1097-1117. 


\section{Caption list}

Fig. 1 Schematic illustration of an in-situ loading rig for the compaction, compression and recovery of expanded graphite worms.

Fig. 2 Variation in sample height (measured in X-ray projections), representing the compaction, compression and recovery behavior of expanded graphite worms. Note that loading step 4 corresponds to the stage of flexible graphite, with a density similar to that of commercial products.

Fig. 3 Polygonal expression of the expanded graphite worm shown in (a). The $\mathrm{ZnO}$ marker particles affixed to the surface (shown in (b)) are considered as vertices of the worm, and connected in $3 \mathrm{D}$ by irregular triangles to create a $3 \mathrm{D}$ polygonal model without overlapping or gaps, as shown in (c). The corresponding 3D polygon mesh is shown in (d).

Fig. 4 3D perspective views of an expanded graphite worm, before and after the first compaction: (a) loading step 0, (b) loading step 1. Images (c) and (d) represent virtual cross sections of cross sections A and B, in (a) and (b), respectively.

Fig. 5 Variations in expanded graphite microdisc shape expressed as polygon meshes. Meshes (a) to (n) correspond to loading steps 0 to 13: (a) - (d) the consolidation process, (e) - (k) the compression process, and (l) - (n) the unloading process. Note that the upper and lower surfaces are shown in yellow and blue, respectively.

Fig. 6 Superposed polygon meshes of an expanded graphite microdisc at loading steps 4 (shown in grey) and 10 (shown in yellow), representing thickness reduction and bending during compression.

Fig. 7 Particle displacements shown as arrows superposed on polygon meshes of an expanded graphite microdisc (upward and downward displacements in blue and red, 
respectively): (a) from loading step 0 to 1 , typical of the compaction process; (b) from loading step 6 to 7, typical of the compression process; and (c) from loading step 10 to 11 , typical of the unloading process.

Fig. 8 Strain distributions on lateral virtual cross sections of an expanded graphite microdisc, obtained by tracking marker particles. Specifications of (a), (b) and (c) as in Fig. 7.

Fig. 9 Strain distributions in the thickness (compression) direction, obtained by measuring local microdisc thickness using marker particle pairs: (a) from loading step 6 to 7, typical of the compression process; (b) from loading step 10 to 11 , typical of the unloading process.

Fig. 10 The same mesh as in Fig. 7 (c), viewed from directly above, representing lateral contraction with vertical recovery in displacement.

Fig. 11 Variations in strain in the compression direction, measured by sample height (whole sample); thickness of the microdisc of interest; and the numerical difference between the two curves, expressing an estimation of the contribution of bending to compression and recovery. 


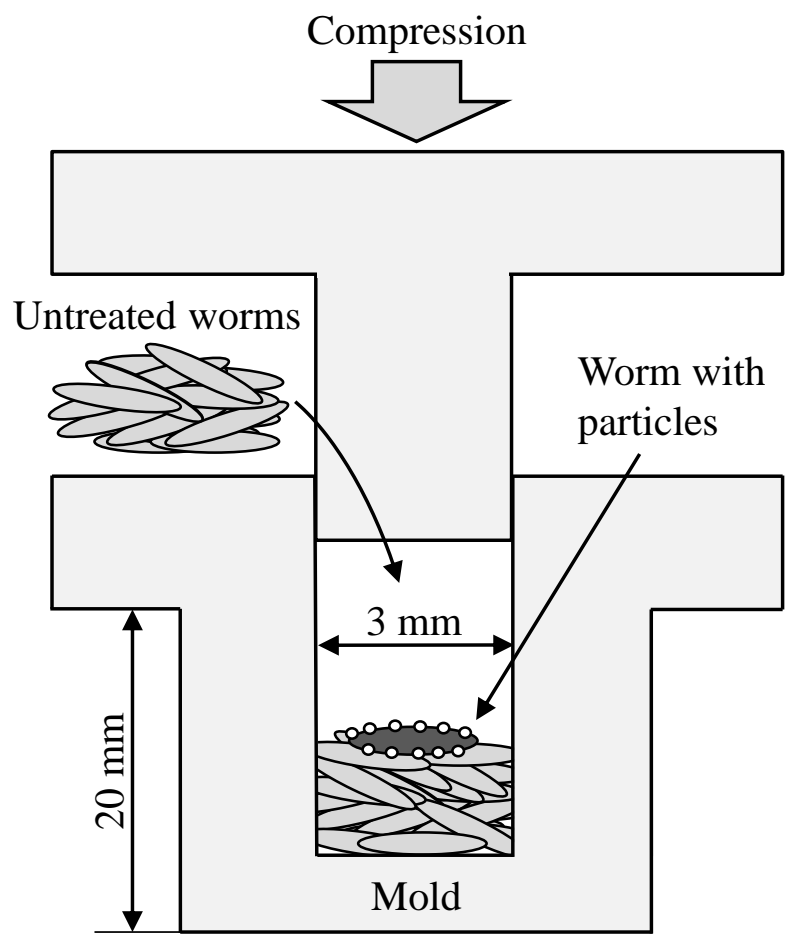

Fig. 1 Schematic illustration of an in-situ loading rig for the compaction, compression and recovery of expanded graphite worms. 


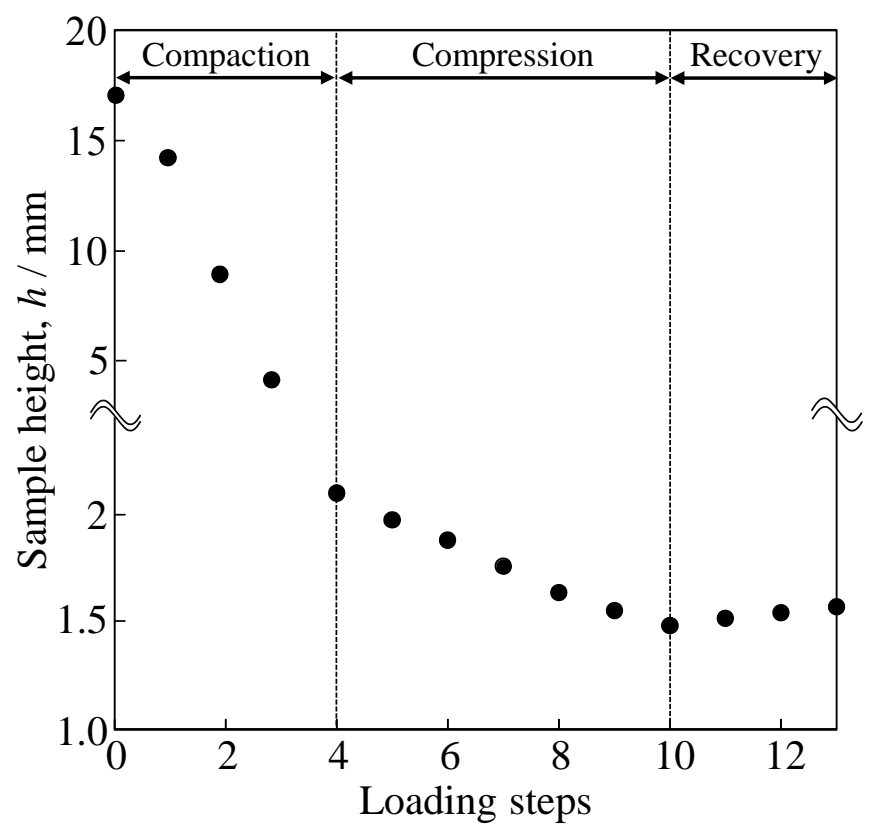

Fig. 2 Variation in sample height (measured in $\mathrm{X}$-ray projections), representing the compaction, compression and recovery behavior of expanded graphite worms. Note that loading step 4 corresponds to the stage of flexible graphite, with a density similar to that of commercial products. 

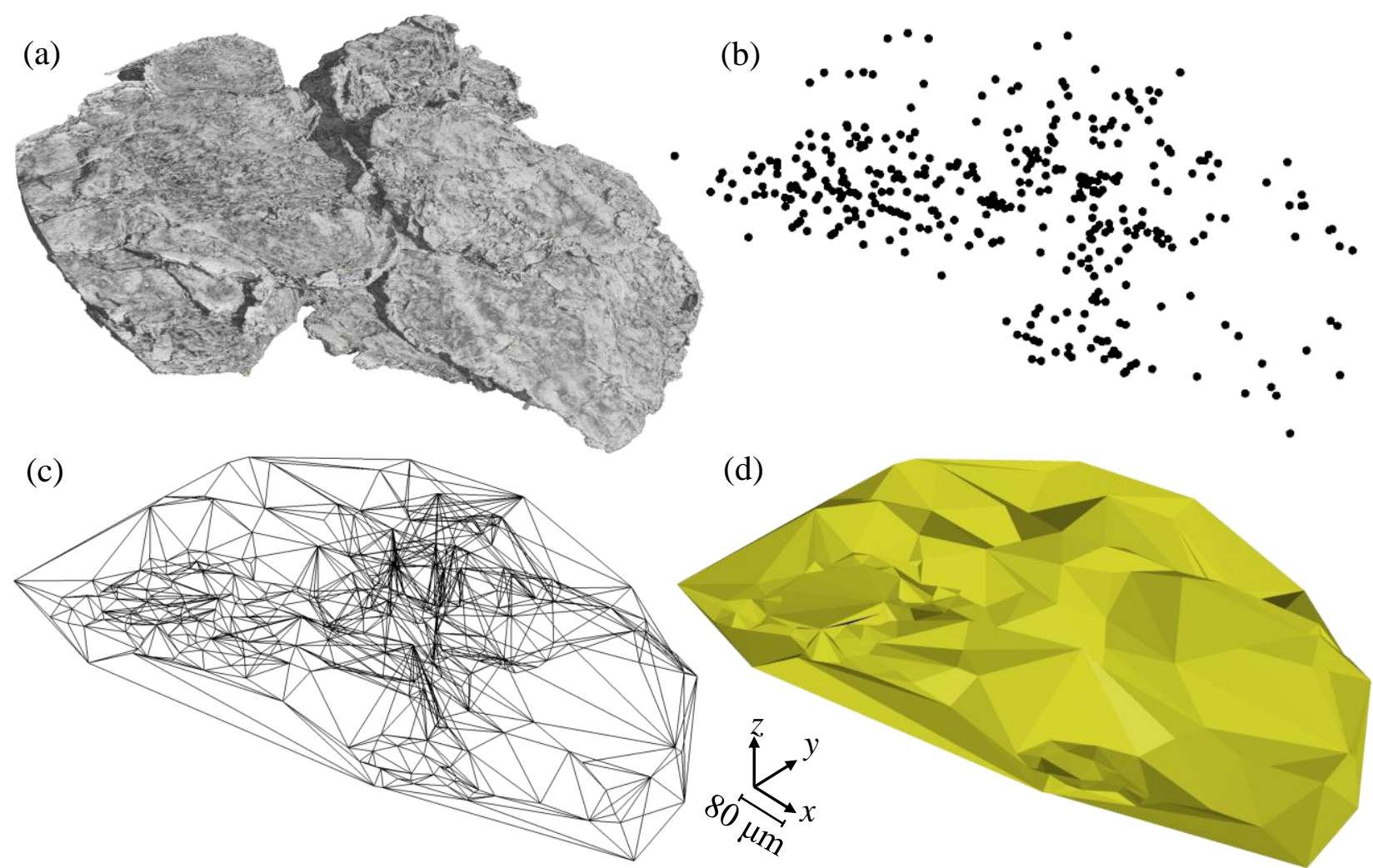

Fig. 3 Polygonal expression of the expanded graphite worm shown in (a). The $\mathrm{ZnO}$ marker particles affixed to the surface (shown in (b)) are considered as vertices of the worm, and connected in 3D by irregular triangles to create a 3D polygonal model without overlapping or gaps, as shown in (c). The corresponding 3D polygon mesh is shown in (d). 

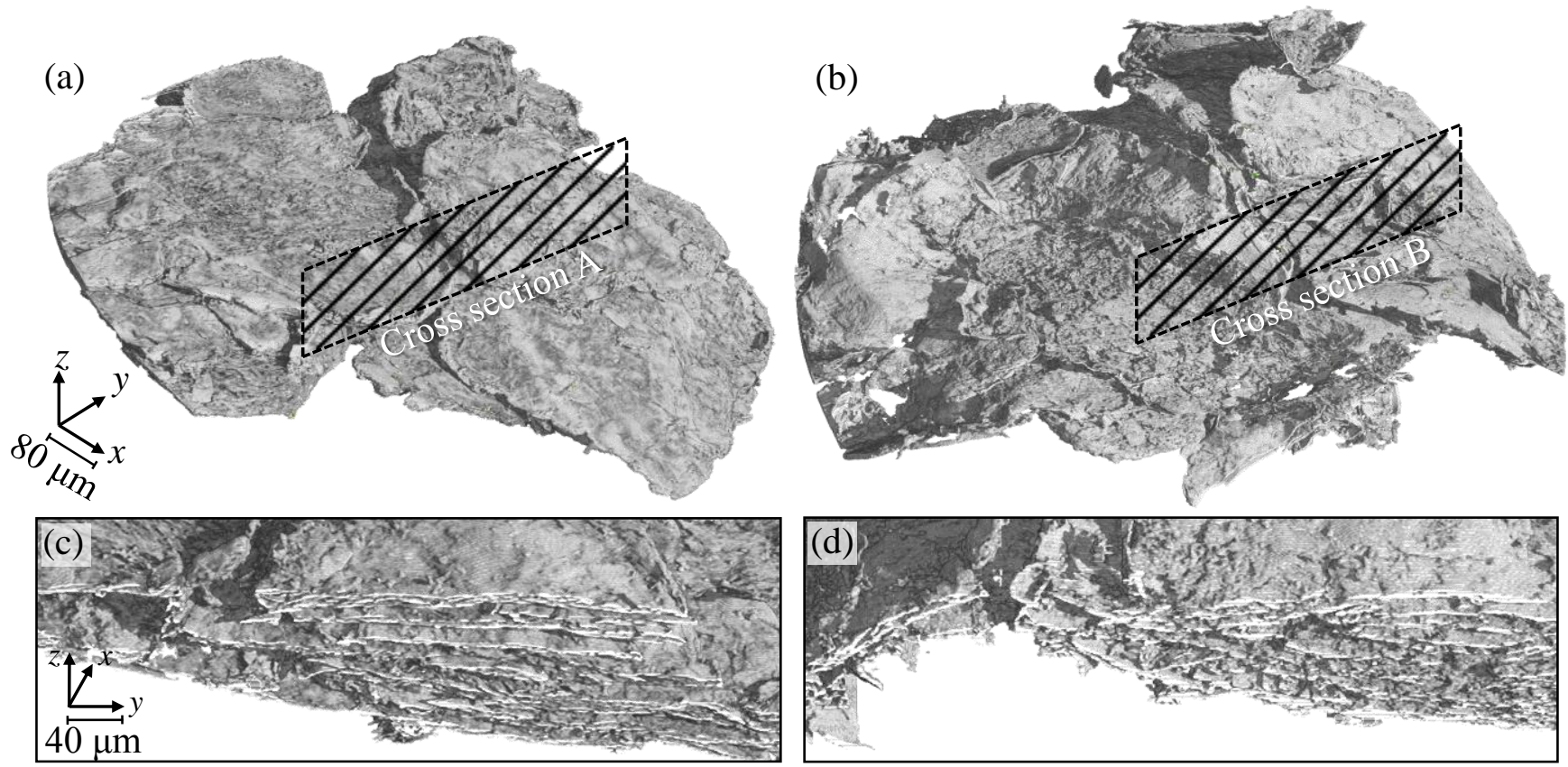

Fig. 4 3D perspective views of an expanded graphite worm, before and after the first compaction: (a) loading step 0, (b) loading step 1. Images (c) and (d) represent virtual cross sections of cross sections A and $\mathrm{B}$, in (a) and (b), respectively. 
(a)
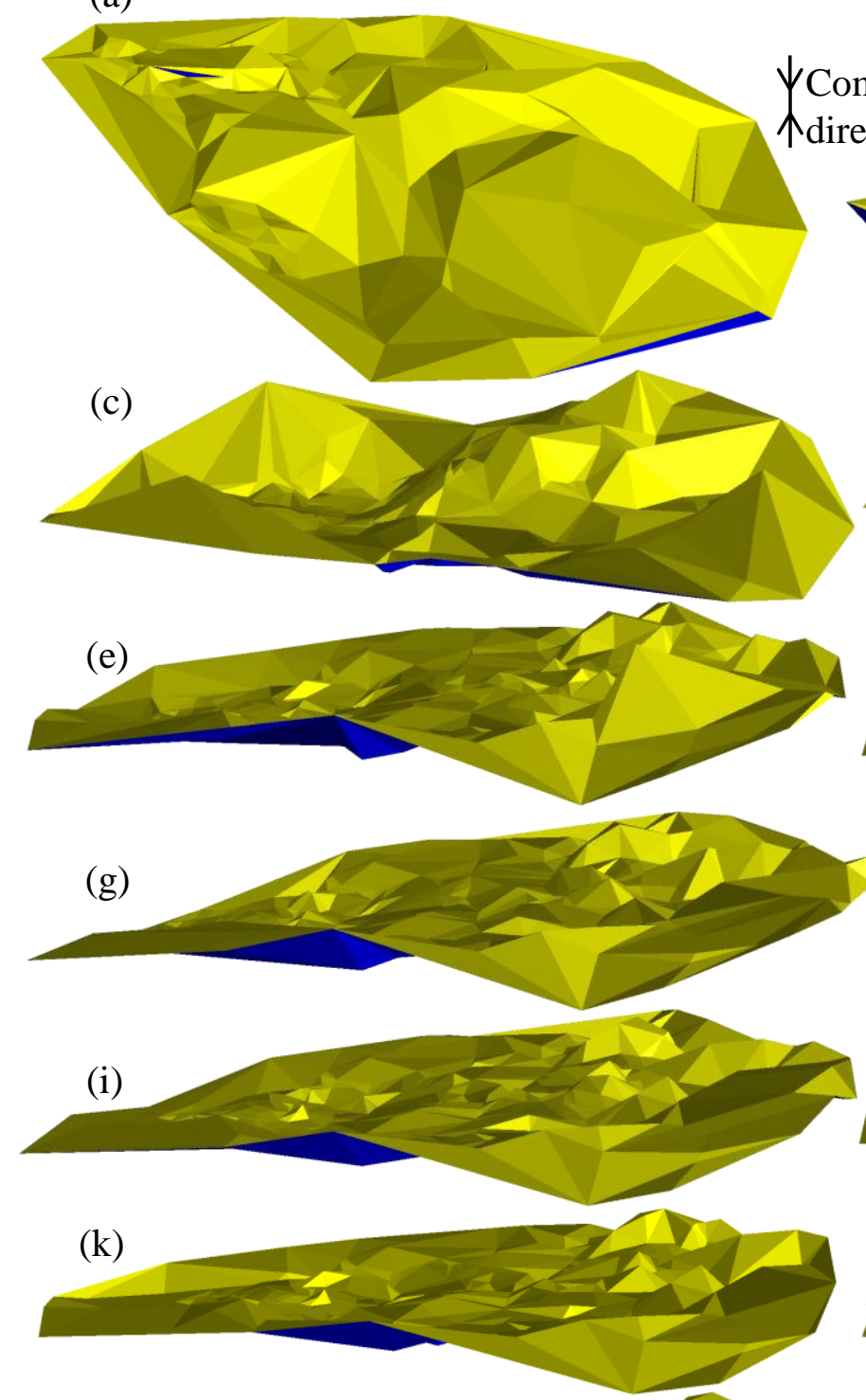

(m)

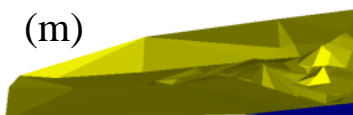

(b)
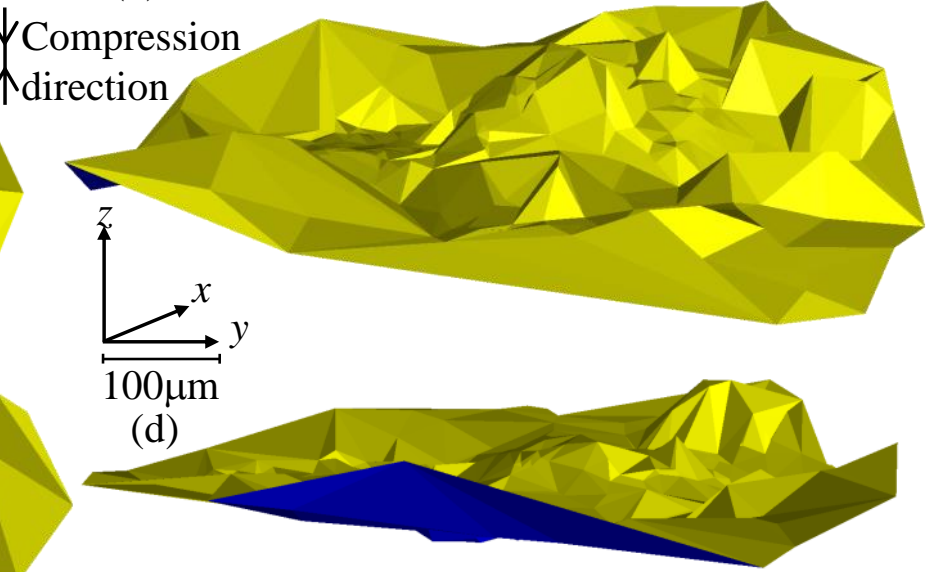

(f)

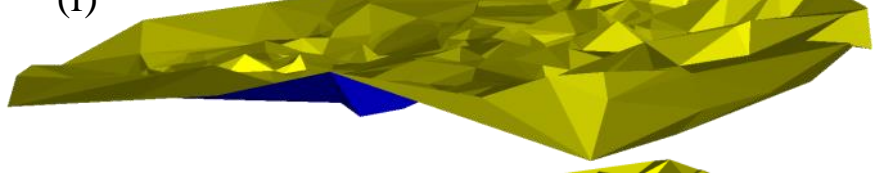

(h)

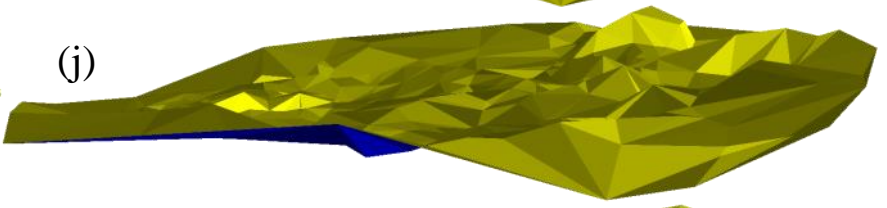

(1)

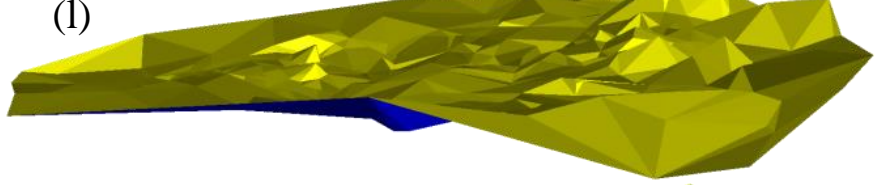

(n)

Fig. 5 Variations in expanded graphite microdisc shape expressed as polygon meshes. Meshes (a) to (n) correspond to loading steps 0 to 13: (a) - (d) the consolidation process, (e) - (k) the compression process, and (l) - (n) the unloading process. Note that the upper and lower surfaces are shown in yellow and blue, respectively. 


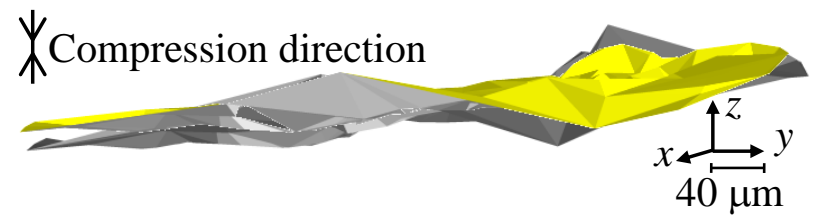

Fig. 6 Superposed polygon meshes of an expanded graphite microdisc at loading steps 4 (shown in grey) and 10 (shown in yellow), representing thickness reduction and bending during compression. 

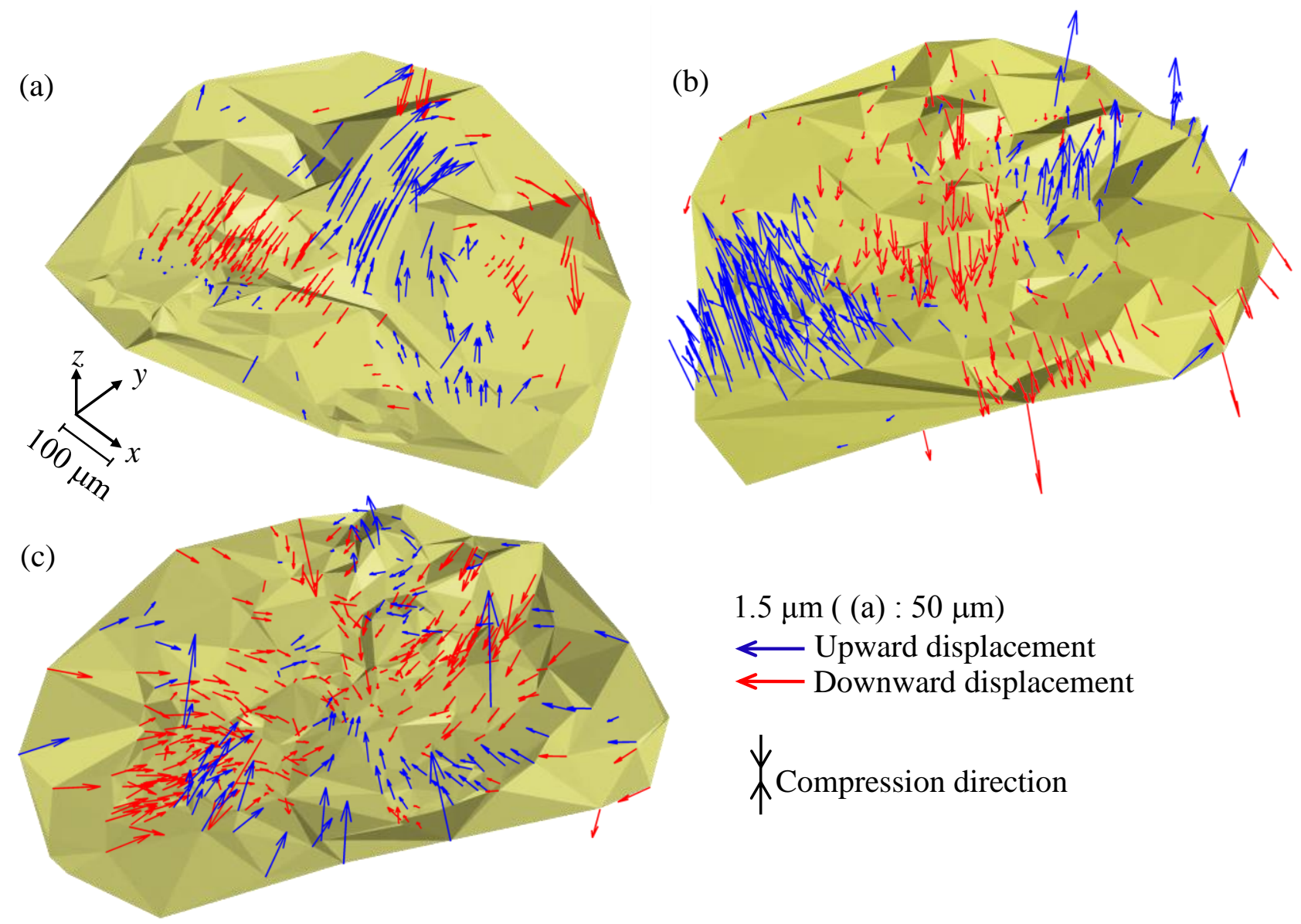

Fig. 7 Particle displacements shown as arrows superposed on polygon meshes of an expanded graphite microdisc (upward and downward displacements in blue and red, respectively): (a) from loading step 0 to 1, typical of the compaction process; (b) from loading step 6 to 7 , typical of the compression process; and (c) from loading step 10 to 11, typical of the unloading process. 


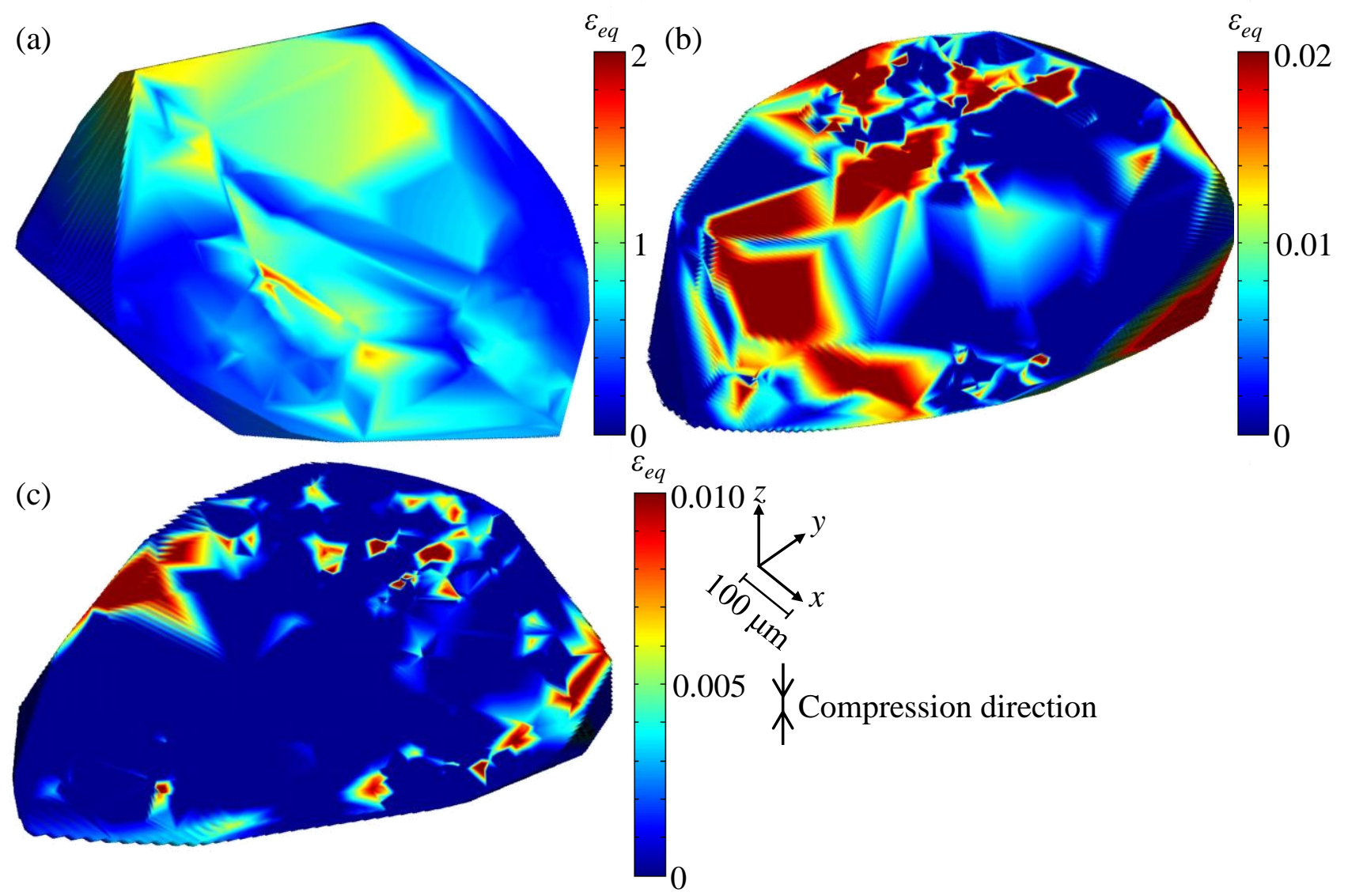

Fig. 8 Strain distributions on lateral virtual cross sections of an expanded graphite microdisc, obtained by tracking marker particles. Specifications of (a), (b) and (c) as in Fig. 7. 


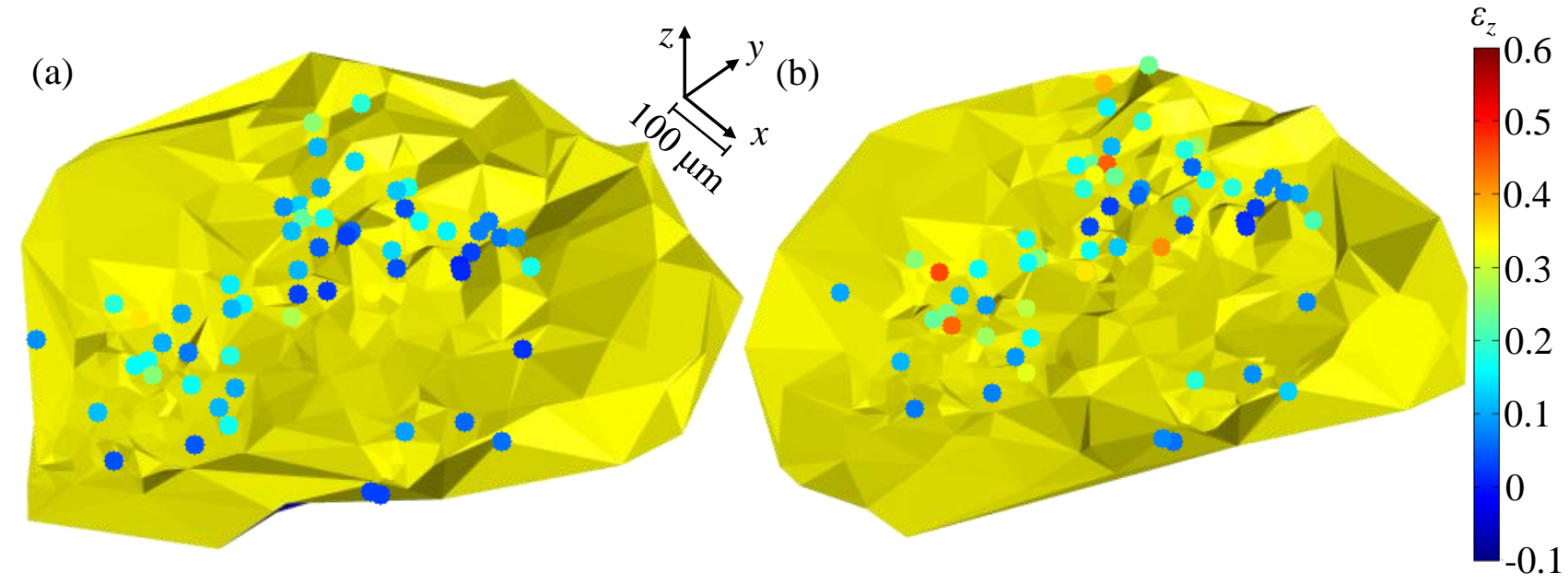

Fig. 9 Strain distributions in the thickness (compression) direction, obtained by measuring local microdisc thickness using marker particle pairs: (a) from loading step 6 to 7 , typical of the compression process; (b) from loading step 10 to 11, typical of the unloading process. 


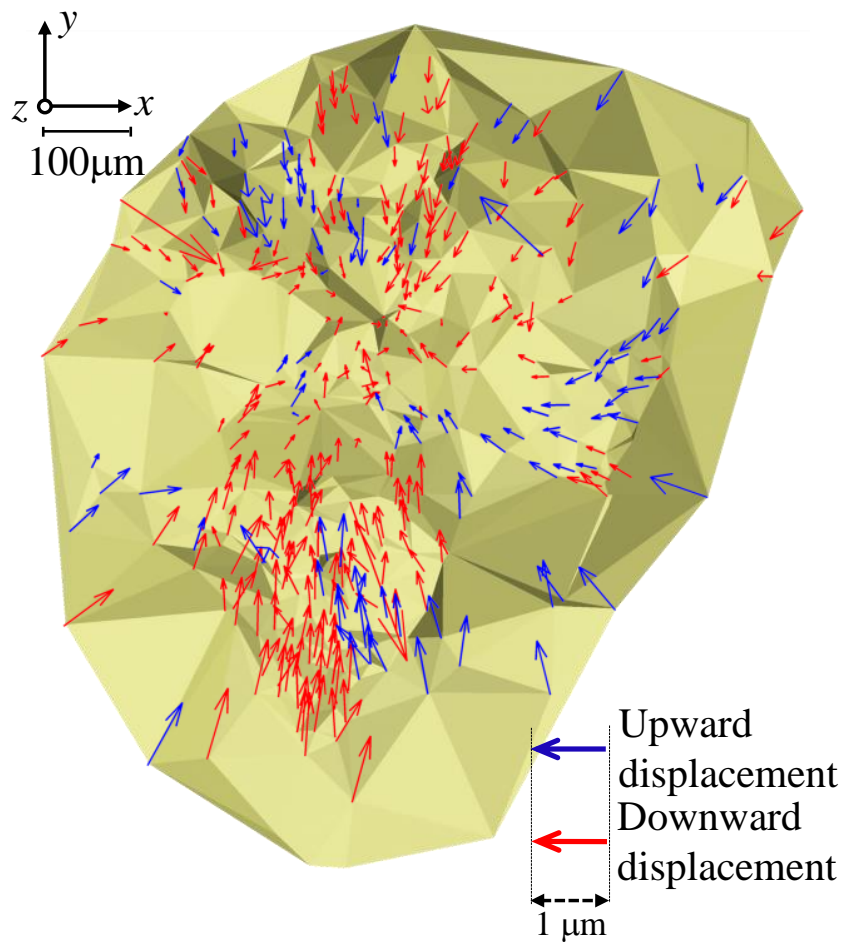

Fig. 10 The same mesh as in Fig. 7 (c), viewed from directly above, representing lateral contraction with vertical recovery in displacement. 


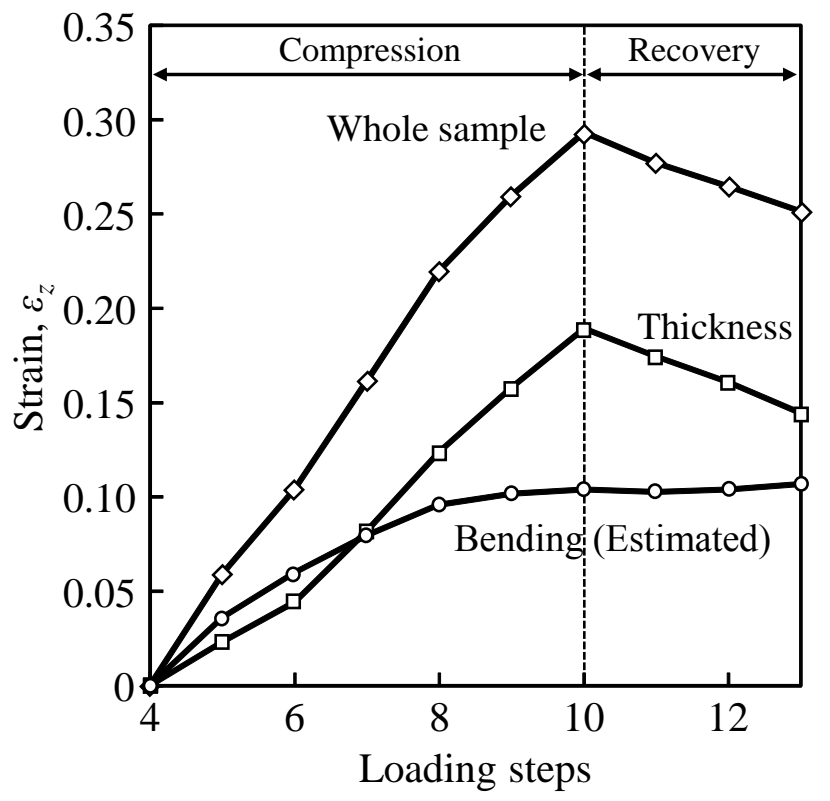

Fig. 11 Variations in strain in the compression direction, measured by sample height (whole sample); thickness of the microdisc of interest; and the numerical difference between the two curves, expressing an estimation of the contribution of bending to compression and recovery. 